\title{
Identification and Prediction of Ionospheric Dynamics Using a Hammerstein-Wiener Model with Radial Basis Functions
}

\author{
Harish J. Palanthandalam-Madapusi, Aaron J. Ridley and Dennis S. Bernstein
}

\begin{abstract}
To construct a model for ionospheric dynamics, a two step identification technique based on subspace algorithms is used. In the first step a Hammerstein model is identified using subspace algorithms and a basis function expansion for the input nonlinearities. In the second step the Wiener nonlinearity is identified as a standard least squares procedure. The inputs to the model are measurements made by the ACE satellite, which is located at the first Lagrangian point between the sun and the earth, while the outputs of the model are ground-based magnetometer readings. To avoid overfitting, the inputs are ranked in order of their effectiveness using an error search algorithm. Results for the ground-based magnetometer located at Thule in Greenland are presented.
\end{abstract}

\section{INTRODUCTION}

Nonlinear identification is an increasingly active research area. Among the various approaches that have been developed are nonparametric methods, which are primarily frequency-domain based. These methods include techniques for identifying Volterra kernels, which characterize inputoutput response by means of a sum of multi-frequency convolutions. For time-domain simulation, however, it is necessary to construct nonlinear state-space realizations of these maps, which may be difficult in practice [16]. On the other hand, parametric methods have been developed based on structured and unstructured time-domain models. Unstructured or black-box models $[8,17]$ often rely on neural network models to exploit their function approximation properties. Structured or grey-box models [4-6,14] are based on the interconnection of linear and nonlinear subsystems.

The most common model structures are the Hammerstein, Wiener, nonlinear feedback, and combined Hammerstein/nonlinear feedback models shown in Figure 1, Figure 2, Figure 3, and Figure 4. These models involve the interconnection of a single linear block and a single nonlinear block. Identification with these model structures has been widely considered, see, for example, $[2,3,7$, 19]. A point that has not been stressed is the fact that nonlinear identification with the Wiener model structure is significantly more difficult than identification with the

H.J. Palanth and D.S.Bernstein are with the department of Aerospace Engineering at the University of Michigan, Ann Arbor, MI 48109-2140. \{hpalanth,dsbaero\}@umich.edu

A.J. Ridley is with the Space Physics Research Laboratory, University of Michigan, Ann Arbor, MI, USA. ridley@umich.edu

This research was supported in part by the National Science Foundation Information Technology Research Initiative, through Grant ATM-0325332.
Hammerstein structure. The reason for this difficulty is the fact that identification of a nonlinear map is more tractable when a measurement of the input to the map is available.

The Hammerstein nonlinear/feedback model can be viewed as a realization of a nonlinear system. Accordingly, the representation of the nonlinearities is not unique [19], and thus it is not possible to uniquely identify the nonlinear maps. For example, the identified maps could differ by a scaling coefficient or a bias.

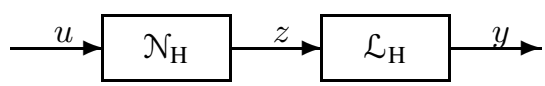

Fig. 1. Hammerstein Model

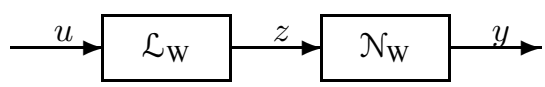

Fig. 2. Wiener Model

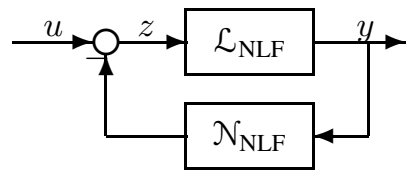

Fig. 3. Nonlinear Feedback Model

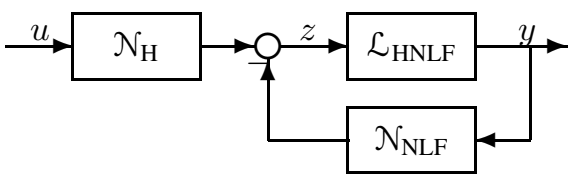

Fig. 4. Hammerstein Nonlinear Feedback Model

In view of the fact that nonlinear identification is more tractable when inputs to the nonlinearity are available, a general formulation of nonlinear identification in this setting was considered in $[9,13]$ using a subspace identification algorithm $[10,18]$ along with a basis expansion for the nonlinear maps. The function expansion was chosen to be linear 
in the parameters, which allows the nonlinear identification problem to be recast as a linear identification problem with generalized inputs. The multivariable capability of subspace identification algorithms is essential to this approach by allowing an arbitrary number of generalized inputs.

In this paper we employ a two step procedure for identifying a Hammerstein-Wiener system for magnetospheric data. In the first step a Hammerstein model is identified using the subspace-based technique developed in $[9,13]$. Next, a static nonlinearity is fitted at the output using a least squares fit. Our approach can be viewed as a variation of two-step nonlinear identification procedures considered previously $[2,15]$.

\section{Magnetospheric Applichtion}

In this paper we apply the nonlinear Hammerstein-Wiener identification approach to measurements of the magnetospheric and ionospheric system. The magnetosphere is the region of space dominated by the magnetic field of the Earth. This region expands out to approximately $10 R_{e}$ (Earth radii) on the day side of the Earth, and many 10's of $R_{e}$ on the night side of the Earth. The size of the magnetosphere and much of the dynamics is controlled by the Sun's atmosphere, which flows super-sonically away from the Sun past the Earth and the other planets. The solar wind carries embedded in it the Sun's magnetic field, commonly referred to as the interplanetary magnetic field (IMF).

The solar wind and IMF interact with the magnetosphere producing phenomena such as the aurora, which occurs in both the northern and southern polar regions. Associated with the aurora are currents. These currents flow along magnetic field lines from the outer magnetosphere to the charged upper atmosphere of the Earth known as the ionosphere. The currents close across the ionosphere.

Most of the time, the aurora and the ionospheric currents are quite calm and have minimal effect on technology. At times, though, the aurora and ionospheric currents can become quite severe. During these time periods, the upper atmosphere can heat up dramatically and expand, causing increased drag on satellites. In addition, the large ionospheric currents can drive currents in power lines, which can overwhelm, and destroy, transformers [12]. It is therefore important that we understand when and where these large currents may occur and be able to predict them.

Ultimately it is the Sun that controls the ionospheric currents and the aurora. When the Sun's atmosphere is calm, there is little aurora and small currents. When there are large ejections of magnetic and plasma energy from the solar surface into interplanetary space, large disturbances in the ionosphere can occur. Satellites monitor the solar surface and measure these events. However, the technology used to model the propagation of these events to the Earth do not have the ability to accurately predict the interplanetary conditions due to disturbances. Therefore, researchers must wait until the ejections reach most of the way to the Earth before measurements are made of their structure. The ACE satellite is positioned at the Lagrangian gravitational null point between the Sun and the Earth. ACE measures the solar wind and IMF conditions approximately one hour before they encounter the magnetosphere. It is therefore the goal of the current study to use the ACE measurements to predict the ionospheric conditions.

Although large-scale, first principles models of the magnetosphere exist, they are extremely expensive to run in real-time, and have to be run at such a poor resolution for real-time performance that the current systems are not well modeled. We therefore turn to empirical models that are simplistic compared to first principles models, but have a tendency to predict specific quantities in the system better than a real-time first principles model.

The ionospheric currents cause magnetic perturbations that can be measured by ground-based magnetometers. We therefore use the magnetometer data to determine the ionospheric conditions. By predicting ground-based magnetometer response using the ACE data, we can determine possible disturbances and may be able to take steps to minimize the economic ramifications.

\section{IDENTIFICATION PROBLEM}

The identification procedure involves a two-step process. In the first step a Hammerstein model is identified using techniques developed in $[9,13]$. Once a Hammerstein model is identified, a static output nonlinearity is identified using a standard least squares fit. Thus, the final identified system is of the form

$$
\begin{aligned}
x_{k+1} & =A x_{k}+F\left(u_{k}\right), \\
v_{k} & =C x_{k}+G\left(u_{k}\right), \\
y_{k} & =\mathcal{N}_{W}\left(v_{k}\right) .
\end{aligned}
$$

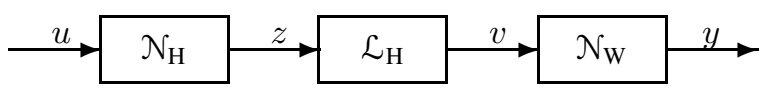

Fig. 5. Hammerstein-Wiener Model

\subsection{Hammerstein Identification}

For the first step of the identification procedure, that is, identification of a Hammerstein model, consider the nonlinear discrete-time system

$$
\begin{aligned}
x_{k+1} & =A x_{k}+F\left(u_{k}\right), \\
v_{k} & =C x_{k}+G\left(u_{k}\right),
\end{aligned}
$$

where $x_{k} \in \mathbb{R}^{n}, u_{k} \in \mathbb{R}^{m}, v_{k} \in \mathbb{R}^{p}, A \in \mathbb{R}^{n \times n}$, $C \in \mathbb{R}^{p \times n}, F: \mathbb{R}^{m} \rightarrow \mathbb{R}^{n}$, and $G: \mathbb{R}^{m} \rightarrow \mathbb{R}^{p}$. The functions $F$ and $G$ can be written in terms of their scalarvalued components as

$$
F(u)=\left[\begin{array}{c}
F_{1}(u) \\
\vdots \\
F_{n}(u)
\end{array}\right], \quad G(u)=\left[\begin{array}{c}
G_{1}(u) \\
\vdots \\
G_{p}(u)
\end{array}\right],
$$


where, for all $i=1, \ldots, n, F_{i}: \mathbb{R}^{m} \rightarrow \mathbb{R}$ and, for all $i=1, \ldots, p, G_{i}: \mathbb{R}^{m} \rightarrow \mathbb{R}$. Defining

$$
z \triangleq \mathcal{N}_{H}(u) \triangleq\left[\begin{array}{l}
F(u) \\
G(u)
\end{array}\right]
$$

the system (3.4), (3.5) can be illustrated as in Figure 1, where $\mathcal{N}_{\mathrm{H}}: \mathbb{R}^{m} \rightarrow \mathbb{R}^{n+p}$ and $\mathcal{L}_{\mathrm{H}}$ represents the linear system

$$
\begin{aligned}
x_{k+1} & =A x_{k}+\left[\begin{array}{cc}
I_{n} & 0
\end{array}\right] z_{k}, \\
v_{k} & =C x_{k}+\left[\begin{array}{ll}
0 & I_{p}
\end{array}\right] z_{k},
\end{aligned}
$$

where $z_{k} \triangleq \mathcal{N}\left(u_{k}\right)$ is viewed as an unmeasured, exogenous input to $\mathcal{L}_{\mathrm{H}}$.

Next, we assume that the components $F_{i}$ and $G_{i}$ can be expanded in terms of a common set of basis functions $h_{1}(u), \ldots, h_{s}(u)$ as

$F(u)=\left[\begin{array}{c}\sum_{i=1}^{s} b_{h 1 i} h_{i}(u) \\ \vdots \\ \sum_{i=1}^{s} b_{h n i} h_{i}(u)\end{array}\right], \quad G(u)=\left[\begin{array}{c}\sum_{i=1}^{s} d_{h 1 i} h_{i}(u) \\ \vdots \\ \sum_{i=1}^{s} d_{h p i} h_{i}(u)\end{array}\right]$.

Defining $h: \mathbb{R}^{m} \rightarrow \mathbb{R}^{s}$ by

$$
h(u)=\left[\begin{array}{c}
h_{1}(u) \\
\vdots \\
h_{s}(u)
\end{array}\right]
$$

it follows from (3.10) that

$$
F(u)=B h(u), \quad G(u)=D h(u),
$$

where $B \triangleq\left[b_{h i j}\right] \in \mathbb{R}^{n \times s}$, and $D \triangleq\left[d_{h i j}\right] \in \mathbb{R}^{p \times s}$. Thus (3.4), (3.5) can be written as

$$
\begin{aligned}
x_{k+1} & =A x_{k}+B h\left(u_{k}\right), \\
v_{k} & =C x_{k}+D h\left(u_{k}\right),
\end{aligned}
$$

The goal of the nonlinear identification problem is to construct models of both $\mathcal{L}_{\mathrm{H}}$ and $\mathcal{N}_{\mathrm{H}}$ given measurements of $\left(u_{k}, v_{k}\right)$ over the interval $0 \leq k \leq \ell$. However, since measurements of $v_{k}$ are unavailable, the signal $y_{k}$ is used for the Hammerstein system identification. The signal $z$ is assumed to be unavailable. However, when $h(u)$ is approximated by $\hat{h}(u)$ and $B, D$ are approximated by $\hat{B}, \hat{D}$, the computed signal

$$
\hat{z} \triangleq\left[\begin{array}{c}
\hat{B} \\
\hat{D}
\end{array}\right] h(u)
$$

is available as the input to $\mathcal{L}_{\mathrm{H}}$.

With the basis functions $h_{i}(u)$ specified, subspace identification algorithms $[10,18]$ can be applied directly to the system (3.12), (3.13) with the computed signal $\hat{z}$ playing the role of the exogenous input.

Two approaches have been developed for choosing basis functions [13]. The selective refinement method involves random generation of collection of basis functions. These basis functions are either retained or discarded depending on the improvement of the cost function. Alternatively, gradient-based optimization algorithms can be used to optimize a set of basis functions with respect to the defining parameters.

\subsection{Wiener Identification}

Once a Hammerstein model has been identified, the output nonlinearity can be estimated by solving a standard linear least squares problem. Let $\hat{v} \in \mathbb{R}^{p}$ be the output of the Hammerstein model. Assuming that the output nonlinearity can be expressed as a basis expansion, a set of $r$ basis functions

$$
f(\hat{v})=\left[\begin{array}{c}
f_{1}(\hat{v}) \\
\vdots \\
f_{r}(\hat{v})
\end{array}\right],
$$

can be used, where $f: \mathbb{R}^{p} \rightarrow \mathbb{R}^{r}$ and $f_{i}: \mathbb{R}^{p} \rightarrow \mathbb{R}$. The above basis functions can either be fixed or can be optimized using a gradient-based scheme. Now, a least squares problem of the form

$$
y=\mathcal{N}_{W}(v) \approx \sum_{i=1}^{r} \lambda_{i} f_{i}(\hat{v})=\Lambda f(\hat{v})
$$

can be considered, where $y$ is the measured data and $\Lambda=$ $\left[\begin{array}{lll}\lambda_{1} & \cdots & \lambda_{r}\end{array}\right]$ is the coefficient matrix. Thus, the least squares solution can be calculated as

$$
\Lambda=y f(\hat{v})^{\mathrm{T}}\left(f(\hat{v}) f(\hat{v})^{\mathrm{T}}\right)^{-1}
$$

\section{IDENTIFICATION FOR MAGnetometer Data}

The ACE IMF and solar wind data are the inputs to the model, while the ground-based magnetometer data are the outputs of the model. Previous studies have shown the relative importance of the components of the IMF and solar wind velocity in driving ionospheric currents. For example, a negative $B_{z}$ component (the $z$-component of the magnetic field) drives strong currents, while a positive $B_{z}$ component drives weak currents. The $B_{y}$ component can drive some currents, while the $B_{x}$ component appears to play a minor role in the magnetospheric dynamics. Therefore, a combination of the $B_{z}$ and $B_{y}$ components is desirable. We have tested several combinations of these two components and find that $B_{t} V_{x} \sin ^{4}(\theta / 2)$ works best, where $B_{t} \triangleq \sqrt{B_{y}^{2}+B_{z}^{2}}, \theta \triangleq \mathrm{a} \cos B_{z} / B_{t}$, and $V_{x}$ is the $x$ component of the velocity of the IMF. Similar combinations of terms have previously been considered [1].

The data used in this study were measured by a magnetometer located at Thule in Northern Greenland. Thule is the highest magnetic-latitude magnetometer. At this location, the ionospheric currents are mostly directly driven by the IMF and the solar wind. At lower latitudes, the currents are sometimes directly driven by the IMF and sometimes driven by the magnetospheric storage and release energy cycle, which complicates the modeling significantly. We plan to model the lower latitude stations in future studies.

At high latitudes, the ionospheric currents form a twocell pattern, flowing toward the Sun across the magnetic pole and away from the Sun at dawn and dusk. A station such as Thule is under the Sunward flow almost all of the time. Because the flow pattern is in a Sun-fixed coordinate system with the station is rotating under it, the system has a 
one-day periodicity. We therefore include a sine wave with a period of one day as a model input.

\section{OVERFITTING DETERMINATION}

Candidate model inputs include $B_{z}, B_{y}, B_{x}$, plasma density, $V_{x}, V_{y}, B_{t}, B_{t} V_{x} \sin ^{4}(\theta / 2)$ and a sine wave with 24-hour periodicity. Since there are several possible sets of inputs to the model, there is a risk of overfitting [11], which generally hampers the predictive capabilities of an empirical model. To avoid overfitting, an error search algorithm is used to rank the inputs in the order of their importance. Specifically, the error search algorithm systematically adds one input at a time and evaluates the prediction error. The prediction error is evaluated as $\sum_{i=j}^{N}(y(i)-\hat{y}(i))^{4}$, where $j$ is the first data point in the prediction region. A quartic error function is used instead of a square function to emphasize the peak finding ability of the model. If the prediction error degrades, that particular input is discarded, even if the fit error improves substantially. This test is performed for both the linearly entering inputs and the nonlinearly entering inputs. A typical plot with the error search algorithm is shown in Figure 6, where each branch in the plot indicates additional complexity in the model in terms of either an additional input or an additional Hammerstein or Wiener nonlinearity. Based on the error search algorithm the most important linearly entering inputs are density, $B_{y}, B_{z}$, and $B_{t}$, whereas the inputs entering through the Hammerstein nonlinearly are $B_{t} V_{x} \sin ^{4}(\theta / 2)$ and the sinusoid with a period of one day.

\section{IDENTIFICATION RESULTS}

The output of the Hammerstein model identified in the first step is shown in Figure 7. Data to the left of the black vertical line are used for identification, and the identified model is used to predict the data to the right of the vertical line. The Hammerstein nonlinear functions in $B_{t} V_{x} \sin ^{4}(\theta / 2)$ and the artificial sinusoid is shown in Figures 8 and 9. Figure 10 shows the output of the complete Hammerstein-Wiener model. Here again only data to the left of the black vertical line is used for identification. A zoomed-in version of the prediction part is shown in Figure 11 , and a plot of the Wiener nonlinearity is seen in Figure 12 .

\section{CONCLUSION}

A two-step identification technique is used to model ionospheric dynamics. In the first step a Hammerstein model is identified using subspace algorithms, and a basis function expansion is used for the input nonlinearities. In the second step the Wiener nonlinearity is identified as a standard least squares step. The inputs to the model are measurements from the ACE satellite, which is located at the first Lagrangian point between the Sun and the Earth, while the outputs of the model are ground-based magnetometer readings. An error search algorithm is employed to rank inputs in order of their importance and to avoid overfitting.
Satisfactory prediction for the ground-based magnetometer at Thule is obtained using the identified model. Future work will focus on other lower-lattitude magnetometers and identification based on richer model structures.

\section{REFERENCES}

[1] S.-I. Akasofu. Predicting geomagnetic storms as a space weather project. In Ling-Hsiao Lyu, editor, Space Weather Study Using Multipoint Techniques, volume 12 of Proceedings of COSPAR, pages 3-20. September 2000.

[2] E.-W. Bai. An Optimal Two-Stage Identification Algorithm for Hammerstein-Wiener Nonlinear Systems. Automatica, 34(3):333338, 1998.

[3] C. H. Chen and S. D. Fassois. Maximum Likelihood Identification of Stochastic Wiener-Hammerstein-Type Non-Linear Systems. Mech. Sys. Sig. Proc., 6(2):135-153, 1992.

[4] H. Diaz and A. A. Desrochers. Modeling of Nonlinear Discrete-Time Systems from Input-Output Data. Automatica, 24:629-641, 1988.

[5] R. Haber and L. Keviczky. Nonlinear System Identification - InputOutput Modeling Approach, volume 1: Nonlinear System Parameter Identification. Kluwer Academic Publishers, 1999.

[6] R. Haber and L. Keviczky. Nonlinear System Identification - InputOutput Modeling Approach, volume 2: Nonlinear System Structure Identification. Kluwer Academic Publishers, 1999.

[7] Z. Hasiewicz. Identification of a Linear System Observed Through Zero-Memory Non-Linearity. Int. J. Sys. Sci., 18(9):1595-1607, 1987.

[8] A. Juditsky et al. Nonlinear Black-Box Models in System Identification: Mathematical Foundations. Automatica, 31(12):1725-1750, 1995.

[9] S. L. Lacy and D. S. Bernstein. Subspace Identification for Nonlinear Systems That Are Linear in Unmeasured States. In Proc. Conf. Dec. Contr., pages 3518-3523, Orlando, Florida, December 2001.

[10] M. Moonen, B. De Moor, L. Vandenberghe, and J. Vandewalle. Onand Off-Line Identification of Linear State-Space Models. Int. J. Contr., 49(1):219-232, 1989.

[11] O. Nelles. Nonlinear System Identification. Springer, 2001.

[12] S. F. Odenwald. The 23rd Cycle. Columbia University Press, February 2001.

[13] H. Palanthandalam-Madapusi, J.B. Hoagg, and D. S. Bernstein. Basis-function optimization for subspace-based nonlinear identification of systems with measured-input nonlinearities. In Proc. Amer. Contr. Conf., Boston, MA, July 2004.

[14] R. K. Pearson. Discrete-Time Dynamic Models. Oxford University Press, 1999.

[15] J. Schoukens, J. G. Nemeth, P. Crama, Y. Rolain, and R. Pintelon. Fast approximate Identification of Nonlinear Systems. Automatica, 39:1267-1274, 2003.

[16] C. A. Schwarz and B. W. Dickinson. On Finite Dimensional Realization Theory of Discrete Time Nonlinear Systems. Sys. Contr. Lett., 7:117-123, 1986.

[17] J. Sjoberg et al. Nonlinear Black-Box Modeling in System Identification: A Unified Overview. Automatica, 31(12):1691-1724, 1995.

[18] P. Van Overschee and B. De Moor. Subspace Identification for Linear Systems: Theory, Implementation, Applications. Kluwer, 1996.

[19] T. Van Pelt and D. S. Bernstein. Nonlinear System Identification Using Hammerstein and Nonlinear Feedback Models with Piecewise Linear Static Maps. Int. J. Contr., 74:1807-1823, 2001. 


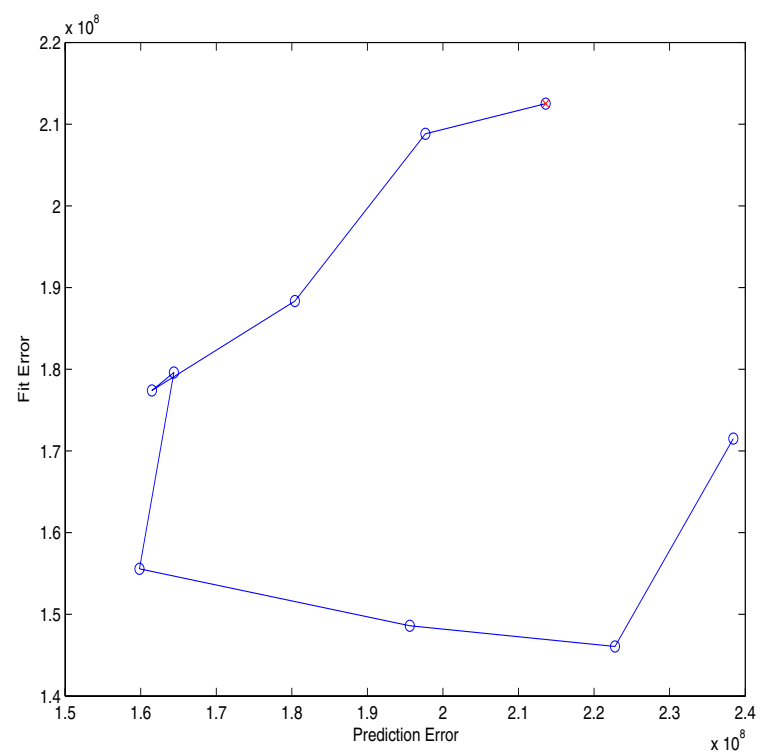

Fig. 6. Illustration of the error search algorithm. Each branch in the plot represents an additional input or nonlinearity in the model. Starting from the point marked with $\times$, in the first few branches the fit error and prediction error are both decreasing, but then the prediction error increases, indicating overfitting.

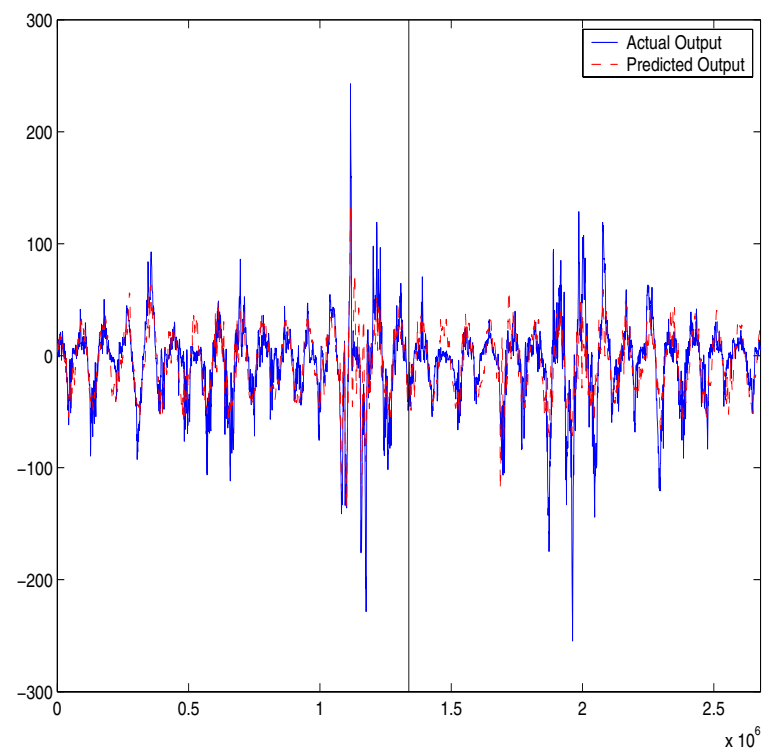

Fig. 7. Measured and predicted data for the Thule magnetometer using the Hammerstein model identified in the first step. Data to the left of the vertical line are used for identification, and data to the right of the vertical line is used to assess prediction using the identified model.

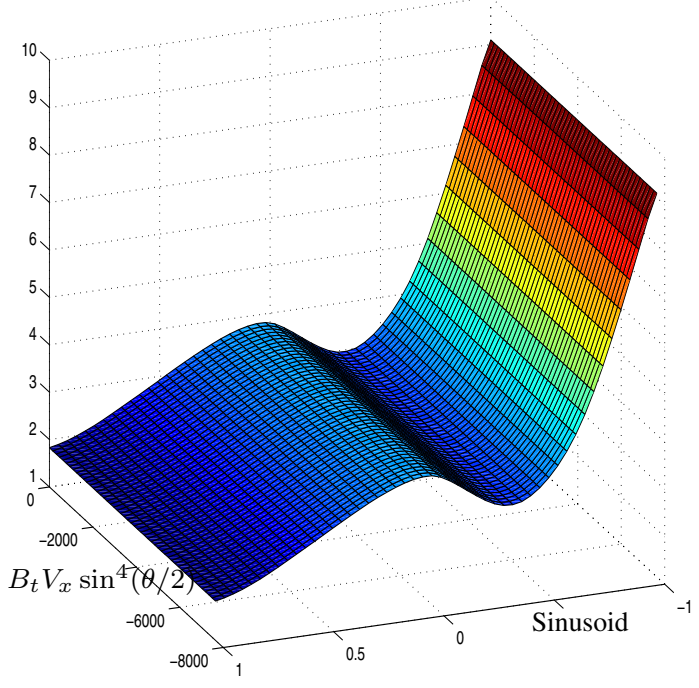

Fig. 8. First dominant Hammerstein nonlinearity $F_{1}(u)$ in $B_{t} V_{x} \sin ^{4}(\theta / 2)$ and a sinusoid with a 24 hour periodicity.

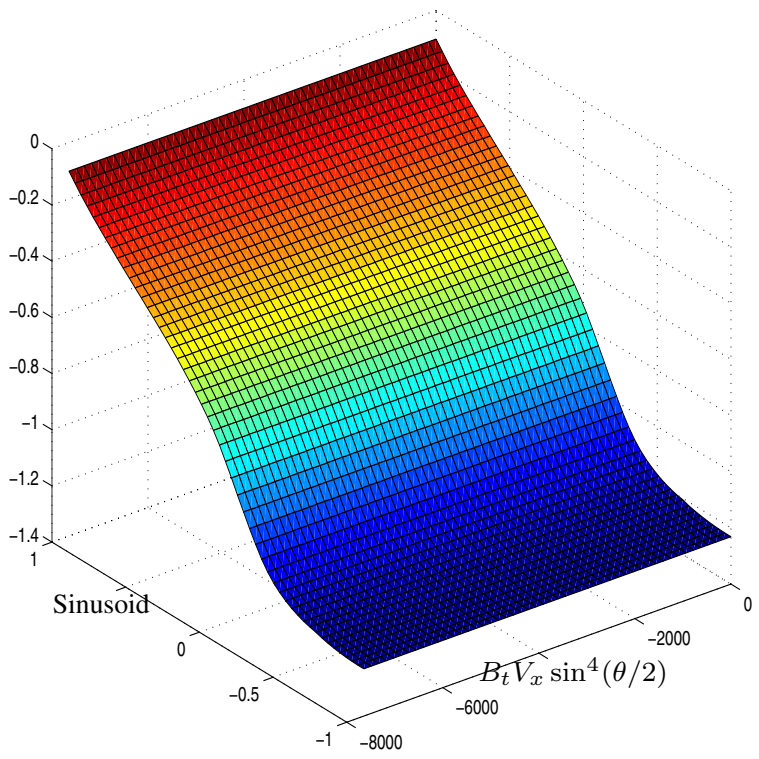

Fig. 9. Second dominant Hammerstein nonlinearity $F_{2}(u)$ in $B_{t} V_{x} \sin ^{4}(\theta / 2)$ and a sinusoid with 24 hour perodicity 


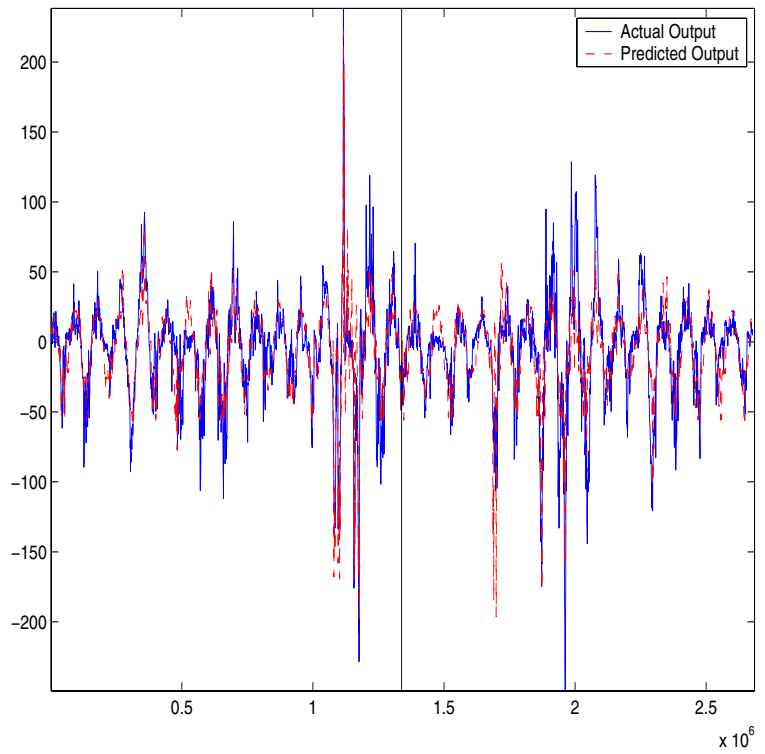

Fig. 10. Measured and predicted data of the Thule magnetometer using a Hammerstein-Wiener model identified in the second step. Data to the left of the vertical line are used for identification, while data to the right of the vertical line are used to assess the prediction ability of the identified model.

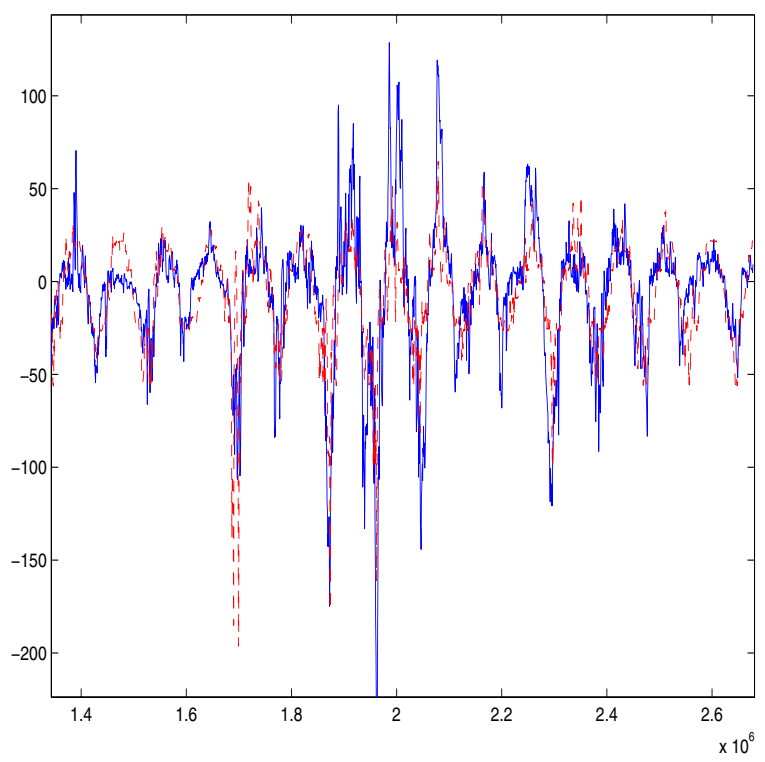

Fig. 11. Magnified view of the prediction region in Figure 10

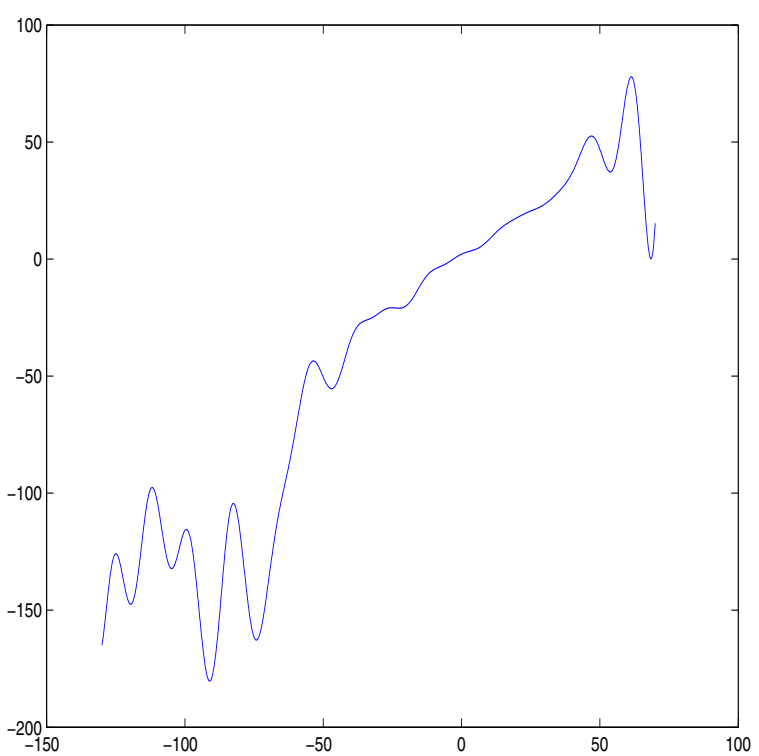

Fig. 12. Wiener nonlinearity $\mathcal{N}_{W}$. After the Hammerstein model identification, the output nonlinearity $\mathcal{N}_{W}$ is estimated using a standard least squares procedure 